Honam Mathematical J. 35 (2013), No. 2, pp. 129-136

http://dx.doi.org/10.5831/HMJ.2013.35.2.129

\title{
ON BOUNDED SOLUTIONS OF PEXIDER-EXPONENTIAL FUNCTIONAL INEQUALITY
}

\author{
Jaeyoung Chung, Chang-Kwon Choi* and Bogeun Lee
}

\begin{abstract}
Let $G$ be a commutative group which is 2-divisible, $\mathbb{R}$ the set of real numbers and $f, g: G \rightarrow \mathbb{R}$. In this article, we investigate bounded solutions of the Pexider-exponential functional inequality $|f(x+y)-f(x) g(y)| \leq \epsilon$ for all $x, y \in G$.
\end{abstract}

\section{Introduction}

Let $G$ be a commutative group which is 2 -divisible, $\mathbb{R}$ the set of real numbers and $\epsilon, \delta \geq 0$. It is well known that if $f: G \rightarrow \mathbb{R}$ satisfies the exponential functional inequality

$$
|f(x+y)-f(x) f(y)| \leq \epsilon
$$

for all $x, y \in G$, then $f$ is an unbounded function satisfying

$$
f(x+y)=f(x) f(y)
$$

for all $x, y \in G$, or a bounded function satisfying

$$
|f(x)| \leq \frac{1+\sqrt{1+4 \epsilon}}{2}
$$

for all $x \in G$ (see Baker[3], Baker-Lawrence-Zorzitto[4]). In particular, if $G=V$, where $V$ is a vector space over the filed $\mathbb{Q}$ of rational numbers

Received February 20, 2013. Accepted March 13, 2013.

2010 Mathematics Subject Classification. 39B82.

Key words and phrases. bounded solution, exponential function, Pexiderexponential functional inequality.

This work was supported by Basic Science Research Program through the National Research Foundation of Korea (NRF) funded by the Ministry of Education, Science and Technology (MEST) (no. 2012008507).

${ }^{*}$ Corresponding author 
and $f: V \rightarrow \mathbb{R}$ is a bounded function satisfying (1.1) for $0 \leq \epsilon \leq \frac{1}{4}$, then $f$ satisfies either

$$
\frac{1+\sqrt{1-4 \epsilon}}{2} \leq f(x) \leq \frac{1+\sqrt{1+4 \epsilon}}{2}
$$

for all $x \in V$, or else

$$
-\epsilon \leq f(x) \leq \frac{1-\sqrt{1-4 \epsilon}}{2}
$$

for all $x \in V$ (see Albert and Baker[2]). It is not easy to describe behavior of bounded solutions when two or more unknown functions are involved in a functional inequality. In this paper, we generalize the result of Albert and Baker[2] and investigate bounded solutions $f, g: G \rightarrow \mathbb{R}$ of the inequality

$$
|f(x+y)-f(x) g(y)| \leq \epsilon
$$

for all $x, y \in G$ under a natural assumption. As a result, we prove the following.

Theorem 1.1. Let $(f, g)$ be a pair of functions satisfying (1.2). Then either $(f, g)$ satisfies

$$
f(x+y)=f(x) g(y)
$$

for all $x, y \in G$, or else $(f, g)$ satisfies

$$
\begin{gathered}
|g(x+y)-g(x) g(y)| \leq k \epsilon, \\
|f(x)-f(0) g(x)| \leq \epsilon
\end{gathered}
$$

for all $x, y \in G$, where

$$
k=\frac{2+M_{g}}{M_{f}}, \quad M_{f}=\sup _{y \in G}|f(y)|, \quad M_{g}=\sup _{y \in G}|g(y)| .
$$

In particular, if $k \epsilon \leq \frac{1}{4}$, then we have

$$
\begin{gathered}
|g(x)-1| \leq \frac{\epsilon}{M_{f}}, \\
|f(x)-f(0)| \leq 2 \epsilon
\end{gathered}
$$

for all $x \in G$. 


\section{Proof of Theorem 1.1}

As a generalization of the result of Albert and Baker[2] we first investigate bounded solutions of the exponential functional inequality

$$
|g(x+y)-g(x) g(y)| \leq \delta
$$

for all $x, y \in G$.

Lemma 2.1. Suppose $0 \leq \delta \leq \frac{1}{4}$ and $g: G \rightarrow \mathbb{R}$ is a bounded function satisfying (2.1). Then, $g$ satisfies either

$$
|g(x)| \leq 2 \delta
$$

for all $x \in G$, or

$$
|g(x)-1| \leq 2 \delta
$$

for all $x \in G$.

Proof. Replacing $x$ and $y$ by $\frac{x}{2}$ in (2.1) we have

$$
g(x) \geq g\left(\frac{x}{2}\right)^{2}-\delta \geq-\delta
$$

for all $x \in G$. Let $g(0)=\beta$. Putting $x=y=0$ in (2.1) we have

$$
\left|\beta-\beta^{2}\right| \leq \delta \text {. }
$$

Thus, we have either

$$
\frac{1-\sqrt{1+4 \delta}}{2} \leq \beta \leq \frac{1-\sqrt{1-4 \delta}}{2},
$$

or

$$
\frac{1+\sqrt{1-4 \delta}}{2} \leq \beta \leq \frac{1+\sqrt{1+4 \delta}}{2} .
$$

If (2.5) holds, putting $y=0$ in (2.1) and dividing the result by $1-\beta$ we have

$$
|g(x)| \leq \frac{\delta}{1-\beta} \leq \frac{1-\sqrt{1-4 \delta}}{2} \leq 2 \delta .
$$

This gives (2.2). Now, assume that (2.6) holds. If $g\left(x_{0}\right) \leq 0$ for some $x_{0} \in G$, putting $x=x_{0}, y=-x_{0}$ in (2.1) we have

$$
\frac{1}{4}=\frac{1}{2}-\frac{1}{4} \leq \beta-\delta \leq g\left(x_{0}\right) g\left(-x_{0}\right),
$$

which implies $g\left(-x_{0}\right) \leq 0$. Thus, from (2.4) we have

$$
-\delta \leq g\left(x_{0}\right) \leq 0, \quad-\delta \leq g\left(-x_{0}\right) \leq 0 .
$$


Thus, we have the contradiction

$$
\frac{1}{4} \leq g\left(x_{0}\right) g\left(-x_{0}\right) \leq \delta^{2} \leq \frac{1}{16} .
$$

Thus, we have $g(x)>0$ for all $x \in G$. Let $M_{g}:=\sup _{x \in G} g(x)$. Replacing $y$ by $y-x$ in (2.1) and using the result we have

$$
g(x) \geq \frac{g(y)-\delta}{g(y-x)} \geq \frac{g(y)-\delta}{M_{g}}
$$

for all $x, y \in G$ such that $g(y)-\delta \geq 0$. Let $K=\{y \in G: g(y) \geq \delta\}$. Then, from (2.7) we have

$$
g(x) \geq \sup _{y \in K}\left(\frac{g(y)-\delta}{M_{g}}\right)=\sup _{y \in G}\left(\frac{g(y)-\delta}{M_{g}}\right)=\frac{M_{g}-\delta}{M_{g}}
$$

for all $x \in G$. Taking the supremum of the left hand side of (2.8) and multiplying both sides of the result by $M_{g}$ we have

$$
M_{g}^{2}-M_{g}+\delta \geq 0 .
$$

Since $M_{g} \geq \beta \geq \frac{1}{2}$, it follows from (2.9) that

$$
M_{g} \geq \frac{1+\sqrt{1-4 \delta}}{2} .
$$

From (2.8) and (2.10) we have

$$
g(x) \geq 1-\frac{\delta}{M_{g}} \geq \frac{1+\sqrt{1-4 \delta}}{2}
$$

for all $x \in G$. Finally, it is well known in [4] that every bounded solution of (2.1) satisfies the inequality

$$
g(x) \leq \frac{1+\sqrt{1+4 \delta}}{2}
$$

for all $x \in G$. Thus, from (2.11) and (2.12) we have

$$
-2 \delta \leq \frac{-1+\sqrt{1-4 \delta}}{2} \leq g(x)-1 \leq \frac{-1+\sqrt{1+4 \delta}}{2} \leq \delta
$$

for all $x \in G$. This gives (2.3). Thus, we complete the proof.

We also use the following result [5].

Lemma 2.2. Let $f, g: G \rightarrow \mathbb{R}$ be bounded functions satisfying the functional inequality

$$
|f(x+y)-f(x) g(y)| \leq \epsilon
$$


for all $x, y \in G$. Then $f, g$ satisfy

$$
|f(x)(1-|g(y)|)| \leq \epsilon
$$

for all $x, y \in G$.

Proof of Theorem 1.1. First, we assume that $g$ is unbounded. Let $y_{n}, n=1,2,3, \ldots$, be a sequence in $G$ such that $\left|g\left(y_{n}\right)\right| \rightarrow \infty$. Putting $y=y_{n}$ in (1.2), dividing the result by $\left|g\left(y_{n}\right)\right|$ and letting $n \rightarrow \infty$ we have

$$
f(x)=\lim _{n \rightarrow \infty} \frac{f\left(x+y_{n}\right)}{g\left(y_{n}\right)}
$$

for all $x \in G$. Multiplying both sides of (2.15) by $g(y)$ and using (1.2) and (2.15) we have

$$
\begin{aligned}
f(x) g(y) & =\lim _{n \rightarrow \infty} \frac{f\left(x+y_{n}\right) g(y)}{g\left(y_{n}\right)} \\
& =\lim _{n \rightarrow \infty} \frac{f\left(x+y+y_{n}\right)}{g\left(y_{n}\right)} \\
& =f(x+y)
\end{aligned}
$$

for all $x, y \in G$. This gives (1.3). Now, we assume that $g$ is bounded. From inequality (1.2) we have

$$
\begin{aligned}
|f(z)||g(x+y)-g(x) g(y)| \leq & |f(z) g(x+y)-f(x+y+z)| \\
& +|f(z+x+y)-f(z+x) g(y)| \\
& +\mid f(z+x) g(y)-f(z) g(x) g(y)) \mid \\
\leq & (2+|g(y)|) \epsilon
\end{aligned}
$$

for all $x, y, z \in G$. It follows from (2.16) that

$$
|g(x+y)-g(x) g(y)| \leq\left(\frac{2+M_{g}}{|f(z)|}\right) \epsilon
$$

for all $x, y, z \in G$. Taking the infimum of the right hand side of (2.17) with respect to $z$ we have

$$
|g(x+y)-g(x) g(y)| \leq\left(\frac{2+M_{g}}{M_{f}}\right) \epsilon
$$

for all $x, y \in G$. Thus, we have (1.4). Now, putting $x=0$ in (1.2) we have (1.5). In particular, if

$$
\delta:=\left(\frac{2+M_{g}}{M_{f}}\right) \epsilon \leq \frac{1}{4},
$$


then by Lemma 2.1, $g$ satisfies either

$$
|g(x)| \leq 2 \delta
$$

for all $x \in G$, or

$$
|g(x)-1| \leq 2 \delta
$$

for all $x \in G$. Assume that $g$ satisfies (2.20). Replacing $y$ by $y-x$ in (1.2) and using the triangle inequality we have

$$
\begin{aligned}
|f(y)| & \leq|f(x) g(y-x)|+\epsilon \\
& \leq 2 \delta|f(x)|+\epsilon \\
& \leq \frac{1}{2} M_{f}+\epsilon
\end{aligned}
$$

for all $y \in G$. Taking the supremum of the left hand side of (2.22) we have

$$
M_{f} \leq 2 \epsilon,
$$

which contradicts (2.19). Therefore, $g$ satisfies (2.21). From (2.19) and (2.21) we have

$$
|g(x)| \leq|1-g(x)|+1 \leq 2 \delta+1 \leq \frac{3}{2}
$$

for all $x \in G$. Thus, from (2.21) and (2.24) we have

$$
|g(x)-1| \leq 2 \delta \leq 2\left(\frac{2+\frac{3}{2}}{M_{f}}\right) \epsilon=\frac{7 \epsilon}{M_{f}}
$$

for all $x \in G$. From (2.19) we have $\frac{7 \epsilon}{M_{f}}<1$. Thus, from (2.25) we have $g(x)>0$ for all $x \in G$. Now, by Lemma 2.2 we have

$$
|f(x)||1-g(y)| \leq \epsilon
$$

for all $x, y \in G$. Thus, we have

$$
|g(x)-1| \leq \frac{\epsilon}{M_{f}}
$$

for all $x \in G$. This gives (1.6). Multiplying (2.26) by $|f(0)|$ we have

$$
|f(0) g(x)-f(0)| \leq \frac{|f(0)|}{M_{f}} \epsilon \leq \epsilon
$$

for all $x \in G$. Putting $x=0$ in (1.2) we have

$$
|f(y)-f(0) g(y)| \leq \epsilon
$$


for all $y \in G$. Using the triangle inequality with (2.27) and (2.28) we have

$$
|f(x)-f(0)| \leq 2 \epsilon
$$

for all $x \in G$. This gives (1.7). Thus, we complete the proof.

Remark 2.3. If $f$ is not extremely small and $g$ is not extremely large as $\epsilon \rightarrow 0$, then $(f, g)$ satisfies the condition (2.19). The opposite case when $f$ is sufficiently small as $\epsilon \rightarrow 0$ can be treated as a trivial case. For example, if $M_{f} / \epsilon$ is bounded as $\epsilon \rightarrow 0$, i.e.,

$$
M_{f} \leq k \epsilon
$$

for some $k>0$ as $\epsilon \rightarrow 0$, then we can easily describe the behavior of $g$ satisfying (1.2). Indeed, using the triangle inequality we have

$$
|f(x) g(y)| \leq|f(x+y)|+\epsilon \leq(1+k) \epsilon
$$

for all $x, y \in G$. Thus, from (2.31) we have

$$
|g(y)| \leq\left(\frac{1+k}{M_{f}}\right) \epsilon
$$

for all $y \in G$. If $f, g$ satisfies (2.30) and (2.32) respectively, then we have

$$
\begin{aligned}
|f(x+y)-f(x) g(y)| & \leq|f(x+y)+| f(x) g(y) \mid \\
& \leq k \epsilon+(1+k) \epsilon=(1+2 k) \epsilon
\end{aligned}
$$

for all $x, y \in G$. Thus, we have

$$
|f(x+y)-f(x) g(y)|=O(\epsilon)
$$

as $\epsilon \rightarrow 0$.

Remark 2.4. We can also find the behavior of $f$ when $g$ is near 0 . Assume that $g$ satisfies

$$
|g(x)| \leq r<1
$$

for all $x \in G$, then replacing $y$ by $y-x$ in (1.2) and using the triangle inequality we have

$$
\begin{aligned}
|f(y)| & \leq|f(x) g(y-x)|+\epsilon \\
& \leq r|f(x)|+\epsilon \\
& \leq r M_{f}+\epsilon
\end{aligned}
$$


for all $y \in G$. Taking the supremum in the left hand side of (2.35) we have

$$
M_{f} \leq \frac{\epsilon}{1-r} .
$$

If $g$ and $f$ satisfy (2.34) and (2.36) respectively, then we have

$$
\begin{aligned}
|f(x+y)-f(x) g(y)| & \leq|f(x+y)|+|f(x)||g(y)| \\
& \leq\left(\frac{1+r}{1-r}\right) \epsilon
\end{aligned}
$$

for all $x, y \in G$.

\section{References}

[1] J. Aczél and J. Dhombres, Functional equations in several variables, Cambridge University Press, New York-Sydney, 1989.

[2] M. Albert and J. A. Baker, Bounded solutions of a functional inequality, Canad. Math. Bull. 25 (1982), 491-495.

[3] J. A. Baker, The stability of cosine functional equation, Proc. Amer. Math. Soc. 80 (1980), 411-416.

[4] J. A. Baker, J. Lawrence, and F. Zorzitto, The stability of the equation $f(x+y)=$ $f(x) f(y)$, Proc. Amer. Math. Soc. 74 (1979), 242-246.

[5] J. Chung, On solutions of exponential functional inequalities, preprint.

[6] D. H. Hyers, G. Isac and Th. M. Rassias, Stability of functional equations in several variables, Birkhauser, 1998.

Jaeyoung Chung

Department of Mathematics, Kunsan National University,

Kunsan 573-701, Korea.

E-mail: jychung@kunsan.ac.kr

Chang-Kwon Choi

Department of Mathematics, Kunsan National University, Kunsan 573-701, Korea.

E-mail: ck38@kunsan.ac.kr

Bogeun Lee

Department of Mathematics, Kunsan National University,

Kunsan 573-701, Korea.

E-mail: lbk@kunsan.ac.kr 